\title{
Effects of practice and signal energy on duration discrimination of brief auditory intervals
}

\author{
THOMAS H. RAMMSAYER \\ University of Giessen, Giessen, Germany
}

\begin{abstract}
In Experiment 1, the proposition that duration discrimination of filled auditory intervals is based on temporal information rather than on energy-dependent cues was tested in 64 naive sub. jects. The subjects were presented with two auditory stimuli at different levels of intensity within one trial, and had to decide which of the two was longer in duration. An adaptive psychophysical procedure was used. As a measure of performance, difference threshold estimates in relation to a 50-msec standard interval were computed. Duration discrimination showed no effect of energy values, indicating that the subjects' discrimination was independent of stimulus intensity. The goal of Experiments 2A and 2B was to investigate the effects of practice on duration discrimination which, in addition, may provide an indirect test for the potential use of energy-dependent cues. Effects of practice on duration discrimination of filled (Experiment $2 \mathrm{~A}$ ) and empty (Experiment $2 \mathrm{~B}$ ) intervals were studied in 6 subjects in each case, over 20 testing sessions. An adaptive psychophysical procedure that was similar to the one used in Experiment 1 was applied. Neither short-term effects of practice based on the first five testing sessions, nor long-term effects of practice based on the means of 4 consecutive weeks, could be demonstrated. The results of the present study suggest that duration discrimination of brief auditory intervals is based on temporal information and not on stimulus energy. Furthermore, implications for the notion of a very basic biological timing mechanism underlying temporal processing of brief auditory intervals in the range of milliseconds are discussed.
\end{abstract}

Quantitative models concerned with the perception of brief time intervals in the range of milliseconds assume that duration discrimination is based exclusively on the temporal extent of the stimuli (Allan, 1979; Allan \& Kristofferson, 1974b; Allan, Kristofferson, \& Wiens, 1971). However, stimulus patterns that differ in temporal extent may also exhibit other differences. Because duration has to be marked by energy signals, it is conceivable that subjects' discrimination between two different durations may be based on some characteristics of the stimuli other than their durations. To be more specific, with filled auditory intervals such as tones or noise bursts, total stimulus energy is proportional to duration and, especially for brief stimuli, loudness is a strong function of duration (Fidell, Pearsons, Grignetti, \& Green, 1970; Garner \& Miller, 1947; Miller, 1948; Munson, 1947; Small, Brandt, \& Cox, 1962). Therefore, discrimination between two auditory intervals in the range of milliseconds may be one of stimulus energy rather than duration discrimination.

On the other hand, experimental data suggest that duration discrimination of filled auditory intervals may be based on an internal time code that is independent of stimulus energy. In a study on duration discrimination, Henry

The author would like to thank Detlev Leutner, Lester Krueger, Lorraine Allan, and two anonymous reviewers for helpful comments. Requests for reprints should be sent to $T$. $H$. Rammsayer, Department of Psychology, University of Giessen, Otto-Behaghel-Str. 10F, D-35394 Giessen, Germany.
(1948) calculated mean Weber ratios for $75 \%$ correct judgments as a function of stimulus intensity. In that experiment, three stimulus sets of $500-\mathrm{Hz}$ tones with base durations of 47,77 , and $277 \mathrm{msec}$ were presented at stimulus levels ranging from 20 to $80 \mathrm{~dB}$. Variation of stimulus intensity had little effect at any stimulus set except for a tendency for a somewhat higher mean Weber ratio for the $20-\mathrm{dB}$ tone in the stimulus sets with base durations of 47 and $77 \mathrm{msec}$. This tendency could be attributed to a decrease in detectability with the low-intensity, shortduration stimuli (Allan \& Kristofferson, 1974b). Furthermore, in a duration-discrimination experiment using the method of constant stimuli, Small and Campbell (1962) presented 47 subjects with four stimulus sets with base durations ranging from 0.4 to $400 \mathrm{msec}$. Changes in Weber ratios observed in this experiment could not be explained in terms of differences in stimulus intensity as a function of duration, because sound-pressure levels (SPL) of the stimuli were adjusted so that all base durations had equal loudnesses. Therefore, the outcome of that study did not support the notion of loudness as a basis for duration discrimination. In a series of experiments employing a signal detection paradigm, Creelman (1962) investigated the effects of stimulus intensity on duration discrimination with filled auditory intervals in the range of milliseconds. Performance in discriminating between a 100- and a 130-msec interval tended to increase with stimulus intensity, but only at low signal-to-noise ratios. When the intervals were made clearly detectable, this effect leveled off and discrimination performance became 
independent of stimulus intensity. Furthermore, on the basis of his results with auditory intervals ranging from 40 to 640 msec, Creelman concluded that "duration discrimination depends on sufficient intensity to mark the time unambiguously; it depends on detectability but not on loudness" (p. 592). Similarly, Abel (1972b) demonstrated that for three sets of noise bursts with base durations of 5,40 , and $320 \mathrm{msec}$, lowpass filtered at $4000 \mathrm{~Hz}$, a decrease in stimulus intensity from 85 to $65 \mathrm{~dB}$ did not affect duration-discrimination performance.

At this point, it appears that duration discrimination of filled auditory intervals in the range of milliseconds can be considered as independent of energy-dependent cues. However, it is important to note that all of these studies suggesting that duration discrimination of auditory intervals is based on the temporal information available in the stimuli rather than on energy-dependent cues provide only indirect evidence for the assumption that duration discrimination is independent of stimulus intensity. This is because the effects of different levels of intensity on discrimination performance were studied by comparing the $\Delta \mathrm{T}$ for $75 \%$ discrimination obtained in separate experimental blocks with the base duration and the comparison intervals always presented at the same level of intensity within each block. Because intensity levels were varied across blocks in these studies (e.g., Abel, 1972b; Creelman, 1962; Henry, 1948), it is not known whether intensity effects would be observed if both the standard and the comparison interval were presented at different intensities within the same trial. To my knowledge, no studies on duration discrimination with auditory intervals exist in which, within one trial, both the standard and the comparison intervals have been presented at different levels of intensity. Therefore, the goal of the first experiment was to elucidate the effect of energy-dependent cues on temporal processing by means of a direct comparison of two auditory intervals presented at different levels of intensity within one trial.

\section{Practice Effects}

In contrast to other areas of perception and psychophysics, very few systematic studies have been designed to explore the effects of practice on duration discrimination of short auditory intervals in the range of milliseconds. In her review on perception of time, Allan (1979) concluded that, for well-practiced subjects, discriminability is independent of base duration over a wide range of duration values. In this respect, the study of practice effects is important because there seems to be a discontinuity of the Weber function with well-trained subjects, whereas with naive subjects, discriminability decreases steadily with increases in base duration (Allan \& Kristofferson, 1974a; Allan et al., 1971; Rousseau \& Kristofferson, 1973). Unfortunately, none of the studies that have reported data from practiced subjects presented sessionby-session performance.

Nevertheless, knowledge about practice-induced changes may not only be helpful for the researcher in- volved in duration-discrimination experiments, but also can help to elucidate mechanisms underlying duration discrimination. In a study on cognitive and perceptual factors involved in duration discrimination, Rammsayer and Lima (1991) provided strong evidence that duration discrimination of brief auditory intervals ranging from 50 to $98 \mathrm{msec}$ is based on processing at the perceptual level, whereas duration discrimination of longer intervals, for instance, those ranging from 1 to $2 \mathrm{sec}$, is cognitively mediated. In a series of experiments, Abel (1972a, 1972b) investigated subjects' ability to discriminate a difference in duration for empty intervals, noise bursts, and gated sinusoids. In Abel's studies, values of the base durations ranged from 0.16 to $960 \mathrm{msec}$. Her results showed that duration discrimination of empty and filled auditory intervals with base durations under $100 \mathrm{msec}$ can be described best by the assumption of an internal clock based on neural counting. Neural counting models such as those originally proposed by Creelman (1962) and Treisman (1963) or, more recently, Church (1984) and Treisman, Faulkner, Naish, and Brogan (1990), which postulate a hypothetical internal clock underlying duration discrimination, may represent a timing mechanism for temporal processing of very brief intervals in the range of milliseconds. According to these accounts, a pacemaker generates pulses, and the number of pulses relating to a time interval is recorded by an accumulator. Thus, the number of pulses counted during a given time interval is the internal representation of this interval. In a two-alternative forced-choice task, a subject is assumed to obtain independent counts and report that the interval having the larger count is longer. Unfortunately, the properties of such a neural timing mechanism are still unknown. Presumably, however, only an internal clock with a stable clock rate could be the basis for exact and highly reliable timing. In this respect, it is important to know to what extent this timing mechanism is affected by practice.

Accordingly, the goal of Experiments $2 \mathrm{~A}$ and $2 \mathrm{~B}$ was to investigate the effects of practice on duration discrimination of filled and empty auditory intervals, respectively. The auditory system tends to integrate signal energy, especially when the stimuli are very short (Fidell et al., 1970; Garner, 1949; Munson, 1947; Small et al., 1962), so differential effects observed with filled and empty intervals may provide an additional indirect test for the potential use of energy-dependent cues.

\section{EXPERIMENT 1}

The purpose of Experiment 1 was to directly test the proposition that temporal discrimination of brief auditory intervals is independent of the energy values of the signals. In previous studies on energy-dependent cues, intensity levels were varied across blocks (e.g., Abel, 1972b; Creelman, 1962; Henry, 1948), but in the present study, both the standard and comparison intervals were presented at different levels of intensity within one trial. Such a direct comparison of two auditory intervals presented at 
different levels of intensity within one trial would represent a more valid evaluation of the effect of energydependent cues on temporal information processing. If duration discrimination is virtually independent of energydependent cues, discrimination performance would not be expected to be significantly different under the following three conditions: (1) the standard and the comparison intervals are of equal loudness, (2) the standard interval is louder than the comparison intervals, and (3) the comparison intervals are louder than the standard interval, and are longer in duration than the standard interval in all three conditions. On the other hand, if duration discrimination is based on differences in stimulus intensity, that is, intervals are judged to be longer simply because they sounded louder, highest performance should be expected for comparison intervals presented at a higher level of intensity than the standard interval, whereas poorest performance should be observed with the standard interval presented at a higher level of intensity than the comparison intervals. On the basis of these considerations, Experiment 1 was designed to gain some more direct evidence for the assumption that duration discrimination of auditory intervals less than $100 \mathrm{msec}$ is independent of stimulus intensity.

\section{Method}

Subjects. The subjects were 40 male and 24 female students ranging in age from 20 to 38 years $(M=25.3 \pm 3.9$ years). All of the subjects had normal hearing and had had no previous experience with temporal discrimination tasks.

Apparatus. The presentation of the auditory duration stimuli and the recording of the subjects' responses was controlled by an IBM XT-compatible computer. Square waves with a frequency of $1000 \mathrm{~Hz}$ were generated by a computer-controlled sound generator.

Design and Stimuli. Three series of intervals were used, consisting of 40 trials each. In the S-C series, both the standard and comparison intervals were presented at a level of $60 \mathrm{~dB}$ (SPL) at the headphones, whereas in the S-CC series, the intensity was $60 \mathrm{~dB}$ (SPL) for the standard interval and $85 \mathrm{~dB}$ (SPL) for the comparison interval. In the SS-C series, the standard and comparison intervals were presented at 85 and $60 \mathrm{~dB}$ (SPL), respectively.

Procedure. The duration stimuli were binaurally presented through headphones (Vivanco Model SR 85). An experimental session consisted of 120 trials. Trials from all three series were randomly alternated. Within each series, the order of presentation for the standard and comparison intervals was randomized and balanced, with each interval presented first in $50 \%$ of the trials. On each trial, two duration stimuli were presented, one standard interval with a constant duration of $50 \mathrm{msec}$ and one variable comparison interval. The initial value of the comparison interval was $98 \mathrm{msec}$. For quantification of performance on duration discrimination, the transformed up-down method was applied, using a two-step rule. Depending on the rule chosen to change the comparison interval, the transformed up-down procedure converges to a specific stimulus value required for a specific level of performance. With the twostep rule, the duration of the comparison interval is decreased after two consecutive correct responses and increased after each incorrect response. Thus, the two-step rule defines the duration of the comparison interval required for $70.7 \%$ correct responses (see Levitt, 1971). Within each series, the duration of the comparison interval changed with a constant step size of $8 \mathrm{msec}$ for Trials 1-10, $4 \mathrm{msec}$ for Trials $11-30$, and 2 msec for Trials 31-50, depending on the subject's previous responses. However, the lower limit of the comparison duration was set at $51 \mathrm{msec}$. Thus, the comparison duration always exceeded the 50 -msec base duration.

The subjects were seated at a table with a computer keyboard and a monitor in a sound-attenuated room. Before the experiment, they were informed that they would be presented with two auditory intervals that would differ in duration in every case, and in intensity in some cases. Furthermore, the instructions emphasized that stimulus duration and intensity might have been integrated by the auditory system and, therefore, a change in either duration or intensity might result in a corresponding alteration in perceived stimulus duration. However, the subjects were told to ignore differences in stimulus intensity and to focus exclusively on stimulus duration. The experimental trials were preceded by nine practice trials consisting of three trials from the S-C, S-CC, and SS-C series, respectively, in which the adaptive procedure was also used. The purpose of the practice trials was to ensure that the subjects understood the instructions and to familiarize them with the stimuli. After the practice trials, the subjects were asked whether they understood the procedure. None of the subjects requested additional practice.

To start a trial, the space bar of the computer keyboard had to be pressed. Then, two auditory intervals were presented with an interstimulus interval of $900 \mathrm{msec}$. The subjects' task was to decide which of the two intervals was longer and to indicate their decisions by pressing one of two designated keys on the keyboard. After each response, visual feedback $(+=$ correct; $-=$ incorrect) was displayed on the monitor screen. The next trial was started by pressing the space bar again.

The subjects were not informed that there was a constant standard interval of $50 \mathrm{msec}$ in every trial. When they were asked after the experiment if they had been aware of different presentation orders of a constant standard and a variable comparison interval, they reported that they had not.

As a measure of performance, mean differences between standard and comparison intervals were computed for the last 20 trials of each testing session. This measure represents an estimate of the individual $70.7 \%$-difference threshold in milliseconds in relation to a standard interval of $50 \mathrm{msec}$. Thus, better performance on duration discrimination is indicated by smaller threshold values.

Data analysis. A one-way analysis of variance (ANOVA) with the series (S-C, SS-C, and S-CC) as three levels of a repeated measures factor was computed for the difference-threshold values. For the within-subjects effects, the Greenhouse-Geisser correction for heterogeneity of variance (Keselman \& Rogan, 1980) was used to determine the appropriate significance level.

\section{Results}

Duration-discrimination performance showed no effect of the energy values of the filled auditory intervals $[F(2,126)=1.16, p=.32]$; the respective $70.7 \%$ difference thresholds were $13.5 \mathrm{msec}$ when both the standard and comparison intervals were presented at a level of $60 \mathrm{~dB}$ (SPL), $14.9 \mathrm{msec}$ when the intensity was $60 \mathrm{~dB}$ (SPL) for the standard and $85 \mathrm{~dB}$ (SPL) for the comparison interval, and $12.4 \mathrm{msec}$ when the standard and the comparison intervals were presented at 85 and $60 \mathrm{~dB}$ (SPL). These results strongly suggest that subjects' discrimination is based only on the temporal extent of the auditory intervals and not on some energy-dependent cues.

A cautionary note, however, is that the finding of intensity invariance in duration-discrimination performance may be due not to a true lack of an effect of stimulus energy, but rather to insufficient sensitivity of the experimental design for measuring the dependent variable. Furthermore, experimental designs that lack sensitivity 
may also result in statistical comparisons that lack power and, correspondingly, cause an overacceptance of the null hypothesis (Lipsey, 1990). Therefore, it must be demonstrated that the null result obtained in the present experiment reflects a genuinely energy-independent process and is not the product of an inadequate experimental design or an insensitive, noisy psychophysical procedure for threshold estimation.

To this end, a power analysis was performed. The power of the statistical test applied in the present study was determined according to the procedure described by Cohen $(1977$, pp. 407-425). To discover a medium effect size of $f^{2}=.15$ with the design used in the present experiment, and given degrees of freedom of the denominator of the $F$ ratio of $v=126$, the resulting noncentrality parameter is $L=18.9$. A significance criterion of $\alpha=.05$ and degrees of freedom of the numerator of the $F$ ratio of $u=2$ yielded a power of .98 for the statistical $F$ test applied. Thus, the outcome of the power analysis indicates that the probability of the statistical test applied in the present study to reject the null hypothesis is .98 . Therefore, the failure to reject the null hypothesis suggests substantial meaning because the a priori probability of rejecting the null hypothesis was high, given the assumption that the effect size is at a medium level.

\section{Discussion}

In the auditory domain, stimulus duration and intensity may be integrated by the hearing mechanism. Therefore, a change in either duration or intensity may result in a corresponding alteration in perceived stimulus magnitude. This effect should be most pronounced at brief durations and low intensities because, under these conditions, decreasing either duration or intensity can lower the integrated stimulus magnitude below the sensation threshold. In this case, the auditory intervals are no longer unambiguously detectable and, therefore, durationdiscrimination performance will necessarily deteriorate. Furthermore, the critical duration above which loudness becomes independent of duration varies as a function of the auditory stimuli applied. Miller (1948) showed that for noise bursts presented at an intensity of $60 \mathrm{~dB}$ above the threshold of audibility, this critical duration was approximately $74 \mathrm{msec}$. A more recent study by Small et al. (1962), also using noise bursts, even suggests a critical duration 2.5 times shorter than that found by Miller (1948). However, critical durations for tones appear to be markedly longer. In a series of experiments, Munson (1947) studied the perceptual growth of auditory sensation using $125-, 1000-$, and $5650-\mathrm{Hz}$ sinusoidal tones presented at several intensities. He found that for $1000-\mathrm{Hz}$ tones presented at $70 \mathrm{~dB}$ (SL), more than $200 \mathrm{msec}$ are required before loudness becomes independent of duration. Similar results are reported by Ekman, B. Berglund, and U. Berglund (1966). Because all durations of the $1000-\mathrm{Hz}$ tones used in Experiment 1 were less than $100 \mathrm{msec}$ and, hence, far below the critical duration be- yond which loudness of tones appears to be independent of duration, energy-dependent cues undoubtedly had a chance to show an effect.

To achieve similar levels of performance under all three experimental conditions, the subjects had to use true temporal discrimination. If intervals were judged to be longer simply because they sounded louder, durationdiscrimination performance should be extremely high for the S-CC series, and markedly poorer for the SS-C series, with performance on the $\mathrm{S}-\mathrm{C}$ series lying between the two. The difference in intensity between the 60 - and 85-dB (SPL) intervals corresponds to a 2.5-fold increase in loudness and was clearly discriminable for all the subjects. Therefore, if intensity were used as a nontemporal cue for duration discriminations-that is, for correct identification of the longer interval-one would expect a probability of hits close to 1 for the S-CC series resulting in extremely small threshold values, because in this series the comparison intervals were always clearly louder than the (shorter) standard interval. On the other hand, threshold values for the SS-C series should be markedly higher than in the S-CC series, because under the SS-C condition the standard interval was clearly louder than the (longer) comparison intervals. In this respect, the outcome of Experiment 1 provides direct support for the proposition that duration discrimination is independent of stimulus intensity.

At least three models may account for the lack of evidence for the potential use of energy-dependent cues in duration discrimination. One of these models is equalization by the hearing mechanism. The persistently low threshold values observed in the present experiment may indicate that the energy integration process of the ear can equalize intensities. The mechanism of such an equalization may well reside in the nonlinearity of the peripheral auditory system. All one has to assume is that an integrator is positioned subsequent to the compressive-nonlinear transduction-an idea that has been proposed by Penner (1975). Furthermore, it may be that the standard interval of the SS-C series possessed an advantage that concealed its true difficulty, namely, that the louder tone was always the constant, $50-\mathrm{msec}$ one. That may have made it a great reference or anchor stimulus, because half of all the loud tones were $50 \mathrm{msec}$ in duration, which may have made them especially salient. The third model that may explain the findings of Experiment 1 is that duration discrimination is based on an internal time code that is independent of stimulus energy. This means that although the auditory system integrates signal energy when the stimulus tones are very short, the internal timing mechanism is not affected by sensory integration. Most models for time perception assume, implicitly or explicitly, that a common timing mechanism underlies duration discrimination in different modalities (Allan, 1979). Therefore, the proposition of a timing mechanism that is not affected by energy-dependent cues would be strengthened if it could be shown that duration discrimination in other mo- 
dalities was also based on the available temporal information, rather than on other cues that may concurrently be available.

Psychophysical studies on visual information processing have also shown that changes in the duration of a brief light flash result in changes in the apparent brightness of the light flash (e.g., J. C. Stevens \& Hall, 1966; S. S. Stevens, 1966). This relationship between luminance and duration is a reciprocal one, as predicted by Talbot's law (Kelly, 1972). Therefore, when subjects are asked to discriminate between brief light flashes differing in duration, their judgments may be based on differences in the apparent brightness of the flashes rather than on their duration. However, Allan et al. (1971) provided experimental evidence that changing the luminance difference between a 100- and a 120-msec light flash did not affect duration-discrimination performance. These results suggest that clearly detectable differences in luminance have not been used as a nontemporal cue for temporal discriminations and that Talbot's law does not hold for duration discrimination in the visual domain. The finding that, for both auditory and visual filled intervals in the range of milliseconds, duration discrimination is independent of the intensity parameters of the stimuli casts some doubt on the proposition of peripheral equalizing effects of the auditory system as a possible explanation for the fact that, in the present experiment, discrimination performance was not affected by stimulus intensity. On the contrary, these results support the notion of an internal timing mechanism that operates only on the temporal information available in the stimulus rather than on other nontemporal cues that may also be present.

\section{EXPERIMENTS 2A AND 2B}

In their visual duration-discrimination experiments in which base durations were 50 and $100 \mathrm{msec}$, Allan et al. (1971) showed that, after extended practice, discriminability was the same for the two base durations. On the other hand, for naive subjects, discriminability was found to be a monotonic decreasing function of stimulus duration. This points to the interpretation that practice may affect duration discrimination in the 50-msec range, at least with filled visual stimuli. Other studies report results consistent with Allan et al. for auditory intervals (see Allan, 1979). However, the major focus of these studies was not on effects of practice and, therefore, no sessionby-session data from experienced subjects were presented.

To my knowledge, the only published experiment primarily concerned with effects of practice on durationdiscrimination performance was a single-subject study by Kristofferson (1980, 1984). Employing a single-stimulus "many-to-few" method (see Allan, 1979) with empty intervals marked by 10 -msec auditory pulses, Kristofferson studied the effect of practice on duration discrimination for 13 stimulus sets with midpoints ranging from 100 to $1,480 \mathrm{msec}$. Difference thresholds indicating performance on duration discrimination over all 13 stimulus sets decreased rapidly during the first six daily sessions consisting of 300 presentations of a single empty interval each, and more slowly thereafter. Estimating the magnitude of the overall effect of practice resulted in a reduction of threshold values of approximately $20 \%$ based on 20 sessions. Unfortunately, no data were provided to evaluate the effects of practice within each stimulus set separately. However, this appears to be a necessary condition because there are different mechanisms underlying duration discrimination, depending on the duration of the stimuli applied (Abel, 1972b; Block, 1990; Rammsayer, 1992b, 1992c).

To date, empirical studies on the effects of practice on duration discrimination with either filled or empty auditory intervals with base durations of less than $100 \mathrm{msec}$ have not appeared in the literature. However, when studying the effects of practice to elucidate the properties of the assumed neural timing mechanism, it is advisable to apply a duration-discrimination task with a base duration under 100 msec because Abel's (1972b) findings suggest that "in both the filled and empty duration experiments, the neural counter model fails in the neighborhood of 100 msec" (p. 1223). Abel's (1972b) conclusion that the countermodel does not provide an adequate description for durations above $100 \mathrm{msec}$ is supported by durationdiscrimination data reported by Getty (1975). Therefore, the major focus of Experiments $2 \mathrm{~A}$ and $2 \mathrm{~B}$ was on the effects of practice on duration discrimination of brief auditory intervals with a base duration of $50 \mathrm{msec}$. The results of these experiments may help to shed some light on the properties of the mechanism underlying duration discrimination of brief auditory intervals in the range of milliseconds.

Although Experiments 2A and 2B were primarily designed to investigate short- and long-term effects of practice on duration-discrimination performance with filled and empty auditory intervals, respectively, these experiments provide an additional indirect test for the use of energy-dependent cues. In filled intervals, physical energy increases as the duration of the interval increases, but in empty intervals, physical energy is constant because empty intervals differ only in the time between the corresponding onset and offset markers (Carbotte \& Kristofferson, 1973). Thus, in empty intervals, no auditory stimulus is presented during the interval itself and, therefore, energydependent cues are not available; in fact, there is virtually no physical energy present. Given that the discrimination of filled intervals may be energy dependent and that of empty intervals presumably is not, if it could be shown that filled intervals are no more susceptible to practice effects than are empty intervals, this would provide useful converging evidence for the claim that discriminability of auditory intervals is independent of the energy values of the stimuli.

\section{Method}

Subjects. Four female and 2 male students ranging from 20 to 37 years of age $(M=27.0 \pm 5.5$ years $)$, and 2 female and 4 male 
students ranging in age from 25 to 36 years $(M=30.2 \pm 4.8$ years) with normal threshold audiograms participated in Experiments $2 \mathrm{~A}$ and $2 \mathrm{~B}$, respectively. All the volunteers were naive subjects who had never participated in any auditory duration-discrimination experiments.

Apparatus and Stimuli. The presentation of the auditory duration stimuli and the recording of subjects' responses was controlled by an IBM XT-compatible computer. Auditory stimuli were generated by a computer-controlled sound generator. In Experiment $2 \mathrm{~A}$, a square wave with a frequency of $1000 \mathrm{~Hz}$ and an intensity of $67 \mathrm{~dB}$ (SPL) was used; in Experiment 2B, the subjects were confronted with empty intervals marked by 3 -msec clicks with an intensity of $88 \mathrm{~dB}$ (SPL). This higher physical intensity, as compared with the filled-interval condition of Experiment $2 \mathrm{~A}$, was necessary to achieve equal loudness in both experiments. This intensity was chosen on the basis of the results of a prior experiment, in which 12 subjects were asked to adjust the loudness of a 3-msec click until it matched that of a 50-msec tone with an intensity of $67 \mathrm{~dB}$ (SPL).

Procedure. The procedure was similar to that of Experiment 1, except that an experimental session consisted of 50 trials. According to the transformed up-down procedure, which converges on a probability of hits of $70.7 \%$, the duration of the comparison interval changed with a constant step size of $8 \mathrm{msec}$ for Trials 1-10, 4 msec for Trials 11-30, and 2 msec for Trials 31-50.

The subjects were instructed to decide which of the two intervals was longer and to indicate their responses by pressing one of two designated keys on the keyboard. They were not informed that there was a constant standard interval of $50 \mathrm{msec}$ in every trial. When asked after the experiment if they had been aware of different presentation orders of a constant standard and a variable comparison interval, they reported that they had not. Each subject was run individually on 20 consecutive daily testing sessions starting on a Monday and omitting weekends; that is, there were 4 weeks of testing of 5 sessions each.

Data analysis. The effects of short-term practice on durationdiscrimination performance were tested by ANOVA with the testing sessions of the first 5-day period as five levels of a repeated measures factor. Long-term practice effects were assessed by ANOVA with the weekly means as four levels of a repeated measures factor. In order to control for the $\alpha$ error, all within-subject effects were subjected to a Greenhouse-Geisser correction.

\section{Results}

Figure 1 displays the results of each subject from Experiment 2A. Performance on duration discrimination with filled auditory intervals is indicated by the $70.7 \%$ difference thresholds in relation to the 50-msec base duration for Sessions 1-20. A repeated measures ANOVA based on Sessions 1-5 did not reveal a statistically significant change in performance on duration discrimination $[F(4,20)=.71, p=.49]$. Thus, there was no evidence of any short-term effect of practice. Similarly, comparison of weekly means of the $70.7 \%$-difference thresholds did not yield statistically significant results $[F(3,15)=.44, p=.60]$, indicating that duration discrimination of short filled auditory intervals was not affected by prolonged practice.

In Figure 2, duration-discrimination performance with empty auditory intervals as a function of session number is presented for each subject of Experiment 2B. A oneway ANOVA, with the first 5 days as five levels of a repeated measures factor, did not reveal a statistically significant increase in duration-discrimination performance $[F(4,20)=1.51, p=.27]$. Likewise, a repeated measures ANOVA based on the weekly means did not yield any statistically significant effects $[F(3,15)=0.22, p=$ .82 ], suggesting that empty auditory intervals of very brief durations are not affected by prolonged practice over 20 testing sessions. Thus, there was no evidence for either a short- or a long-term effect of practice on durationdiscrimination performance with short, empty auditory intervals.

Although no effects of practice could be shown in Experiments $2 \mathrm{~A}$ and $2 \mathrm{~B}$, there obviously was a difference in the overall level of performance with filled (mean difference threshold $=7.1 \mathrm{msec}$ ) as compared with empty intervals (mean difference threshold $=12.5 \mathrm{msec}$ ), indicating that duration discrimination was better with filled than with empty intervals. However, a $t$ test revealed that this difference just failed to reach statistical significance $[t(10)=1.92, p=.08]$.

\section{Discussion}

The results of Experiments 2A and 2B clearly show that performance on duration discrimination with either filled
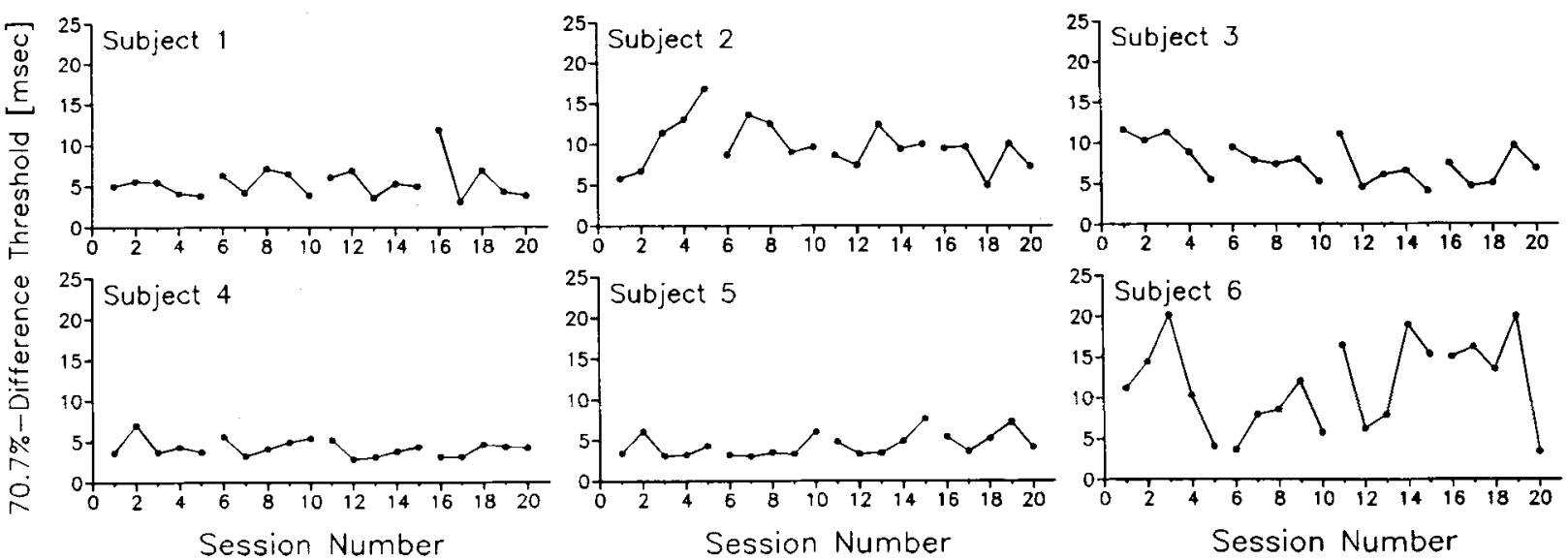

Figure 1. Duration-discrimination performance with filled auditory intervals (Experiment 2A) as a function of session number for each subject. Performance is indicated by $70.7 \%$-difference threshold estimates. Subjects 1, 2, 3, and 6 were females; Subjects 4 and 5 were males. 

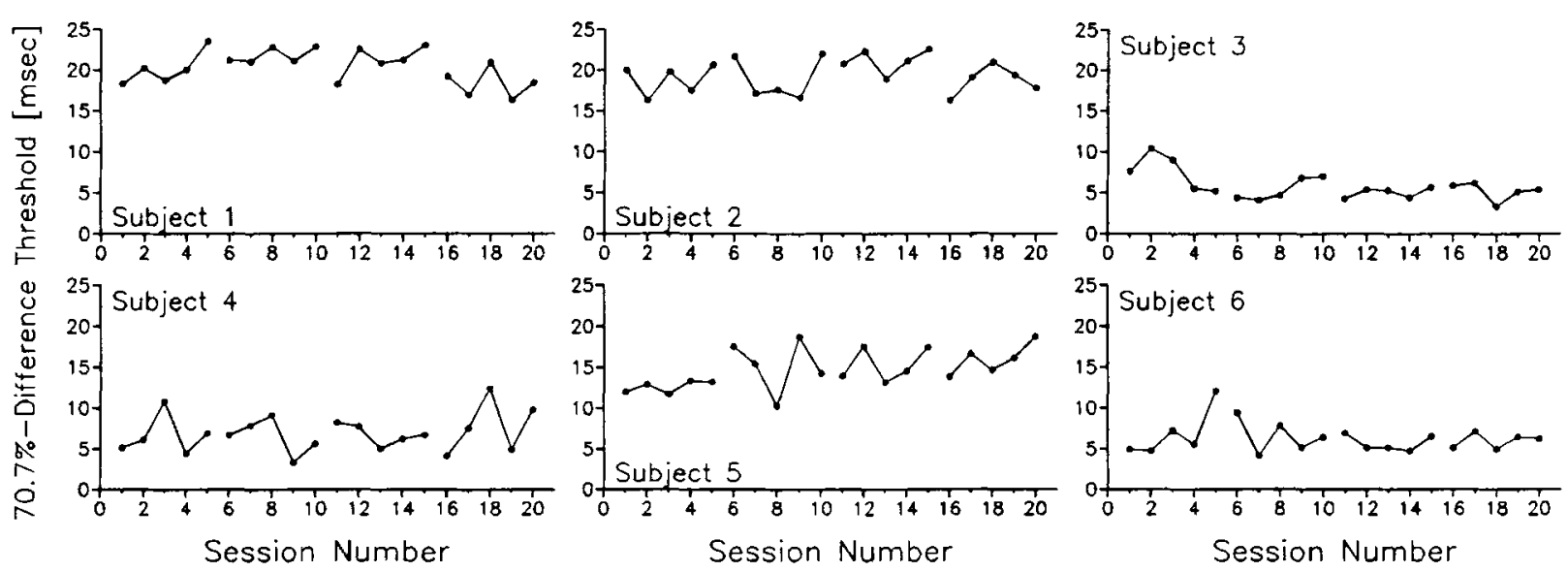

Figure 2. Duration-discrimination performance with empty auditory intervals (Experiment 2B) as a function of session number for each subject. Performance is indicated by $70.7 \%$-difference threshold estimates. Subjects 2 and 3 were females; Subjects $1,4,5$, and 6 were males.

or empty auditory intervals in the range of milliseconds did not improve over 20 testing sessions. A cautionary note, however, is that the finding of no practice effects in Experiments 2A and 2B may be due not to a true lack of a practice effect, but to insufficient sensitivity of the procedure applied. Therefore, it must be demonstrated that the null results obtained with filled and empty intervals reflect genuinely practice-invariant processes and are not the product of an insensitive, noisy psychophysical procedure for threshold estimation. Therefore, in order to evaluate the sensitivity of assessment, Cronbach's alpha (e.g., Cronbach, 1990) was computed across the 20 threshold estimates based on the 20 consecutive daily testing sessions. Reassuringly, the resulting alpha coefficients were .95 and .99 for Experiments $2 \mathrm{~A}$ and $2 \mathrm{~B}$, respectively, indicating that individual differences were highly stable across trials. This high level of internal consistency supports the view that the null results obtained in the present study reflect a true lack of practice effects in duration-discrimination performance rather than insensitivity because of unreliable techniques or instrumentation. It is also important to note that the psychophysical procedure applied in the present experiments is highly sensitive to true individual differences in durationdiscrimination performance. In a study on sex differences, for example, this procedure has yielded results indicating duration-discrimination performance differences between male and female subjects (Rammsayer \& Lustnauer, 1989). Also, this procedure yielded differences in temporal processing among psychiatric patients with different diagnoses (Rammsayer, 1990) and between blind and sighted subjects (Rammsayer, 1992a). Therefore, it is very unlikely that the procedure used in the present study was insufficiently sensitive to detect effects of practice.

When comparing the present experiments with other practice studies (e.g., Allan et al., 1971; Kristofferson, 1980), some potentially important differences become evi- dent. For example, the total amount of practice, in terms of number of trials given in Experiments $2 A$ and $2 B$ was smaller than in other practice studies. In the present study, the subjects were presented with 1,000 trials, whereas there were 6,000 trials in Kristofferson's (1980) singlesubject study and at least 15,000 trials per subject in $\mathrm{Al}$ lan et al.'s study. However, the difference in number of trials is unlikely to account for the absence of practice effects in the present experiments because in Kristofferson's study, "threshold decreases rapidly for the first half dozen sessions and more slowly thereafter' (Kristofferson, 1980, p. 304). The implication is that the most pronounced effect of practice is expected to occur in the early phase of the experiment and, therefore, should have manifested itself in the present study despite the limited number of trials. Furthermore, in the present study, an adaptive psychophysical forced-choice procedure was used in which two stimuli were presented on each trial. One stimulus was always a constant standard interval with a base duration of $\mathbf{5 0} \mathrm{msec}$ and the other stimulus was a variable comparison interval. In contrast, Kristofferson (1980) employed a many-to-few procedure, a modified version of the method of single stimulus. With this procedure the midpoint of a given stimulus set (i.e., the respective base duration) is not presented, but stimuli shorter or longer than the respective midpoint (base duration) are to be categorized as "long" or "short" in relation to the base duration of the stimulus set. Thus, the base duration is assumed to be an internal representation of the midpoint of the stimulus set given. Because the base duration itself is never presented, each subject has to create and somehow store an internal representation of the base duration during the course of the experiment. Therefore, the singlestimulus many-to-few method employed by Kristofferson (1980) may involve other modes of processing than the adaptive psychophysical method used in the present study. In this respect, a comparison of the results reported by Kristofferson (1980) with those of the present Experiments 
$2 \mathrm{~A}$ and $2 \mathrm{~B}$ suggests that the very pronounced effect of practice found in Kristofferson's study may be largely task specific and, therefore, unrelated to the timing process itself.

In the present study, mean Weber fractions obtained at the end of 20 sessions were .10 and .25 for filled (Experiment 2A) and empty (Experiment 2B) intervals, respectively. Weber fractions for both types of intervals concur with findings of previous studies. In a study on duration discrimination of noise bursts and $1000-\mathrm{Hz}$ sinusoids, Abel (1972b) found a mean Weber fraction of .10 for filled intervals with a base duration of $50 \mathrm{msec}$, which is in perfect agreement with the outcome of Experiment 2A. Weber fractions for empty intervals reported by different authors vary considerably and, therefore, are far more equivocal than those reported for filled intervals.

There are at least two critical points that have to be taken into consideration when comparing the Weber fractions of empty intervals from different studies. First, there are procedural discrepancies in how to define the duration of an empty interval. Although some researchers measure intervals from marker onset to marker offset (e.g., Divenyi \& Danner, 1977; Divenyi \& Sachs, 1978), most others define the intervals to be discriminated as those between the offset of the first and the onset of the second marker (e.g., Abel, 1972a; Carbotte \& Kristofferson, 1973; Kristofferson, 1980, 1984; Penner, 1976; Rammsayer \& Lima, 1991). To control for such procedural discrepancies is crucial, especially when long marker durations in combination with relatively brief base durations are used because, otherwise, the former definition would lead to a systematic underestimation of the Weber fraction as compared with the latter one.

Second, the results of Experiment 2B show that with an unselected sample of subjects, there are very pronounced interindividual differences in levels of performance on duration discrimination, as indicated by individual threshold values (see Figure 2). In most duration-discrimination experiments, very few subjects have been studied and, therefore, selection of subjects becomes a critical issue. For example, in a series of experiments with empty auditory intervals, Divenyi and Danner (1977, Experiments 1 and 3) obtained Weber fractions averaging approximately .085 for a base duration of $60 \mathrm{msec}$ (measured from the onset of the first marker to the offset of the second marker). However, these data are based on 4 subjects who were "extremely capable" and who were "selected from a pool of seven subjects who had received several months of previous training' (Divenyi \& Danner, 1977, p. 127). On the other hand, Abel (1972a) reported a mean Weber fraction of .57 for a base duration of $40 \mathrm{msec}$ based on 3 subjects. Unlike Divenyi and Danner, Abel studied 1 experienced subject and 2 experimentally naive subjects. Therefore, their performance levels for this type of task could not have been known in advance. The marked difference in Weber fractions between Abel's (1972a) and Divenyi and Danner's studies may be explained in terms of practice effects, because Divenyi and Danner used only highly trained subjects. However, the absence of practice-induced changes in duration discrimination with empty intervals in combination with very pronounced and persistent interindividual differences, as observed in Experiment $2 \mathrm{~B}$, may point to the alternative interpretation that selection of subjects may also account for the discrepancies found when comparing Weber fractions reported from studies by different authors. In the present study, individual Weber fractions for empty intervals obtained with an unselected sample varied from .11 to .41 , which is well within the expected range of variation based on the results of Divenyi and Danner, representing the lower limit, and on those of Abel (1972a), representing the upper limit.

\section{GENERAL DISCUSSION}

The results of Experiment 1 provide some direct evidence for the proposition that duration discrimination of auditory filled intervals of less than $100 \mathrm{msec}$ is not affected by changes in signal energy. In addition, Experiments $2 \mathrm{~A}$ and $2 \mathrm{~B}$ provide an indirect test for the potential use of energy-dependent cues, which comes up empty handed by showing that filled intervals, whose discrimination may be based on the potential use of energydependent cues, are no more susceptible to practice effects than are empty intervals, whose discrimination is not energy dependent (Carbotte \& Kristofferson, 1973). In filled intervals, a tone or noise burst is presented continuously throughout the interval, whereas in empty intervals only the onset and the offset of the interval are marked by clicks. Therefore, empty intervals differ in temporal extent only, and there are no cues related to the total energy of the stimuli.

The lower overall level of performance with empty intervals in Experiment $2 \mathrm{~B}$ as compared with filled intervals in Experiment $2 \mathrm{~A}$ is in agreement with previous findings in which duration-discrimination performance on filled auditory intervals was better than on empty auditory intervals (Abel, 1972a, 1972b; Allan, 1979; Craig, 1973; Rammsayer \& Lima, 1991). However, as long as the mechanisms underlying temporal processing of filled and empty intervals are not known precisely, the fact that filled intervals are more readily discriminated than empty intervals may still point to the potential use of energydependent cues in the case of filled intervals.

In a series of experiments done with a durationdiscrimination paradigm identical to the one used in the present study, Rammsayer and Lima (1991) demonstrated that superior performance on filled intervals as opposed to empty intervals is not due to a difference in cognitive demands, but to a difference in perceptual processing. Although the ultimate reason for better durationdiscrimination performance with filled than with empty auditory intervals is not yet completely understood, Rammsayer and Lima presented several possible expla- 
nations for the differences in processing filled and empty intervals, which are based on notions of perceptual processing.

According to the process model of timing described by Church (1984), the internal timing mechanism is composed of a pacemaker, a switch, and an accumulator. The pacemaker generates pulses that are switched into the accumulator. On the basis of animal studies, Church concluded that the switch could be operated in different modes, some much more complex than others. It is plausible to assume that the simplest switch mode, in which the switch is on at the onset of a signal and off at the offset of a signal, was applied to filled-interval trials (Experiment $2 \mathrm{~A}$ ), whereas a more complex mode was applied to the empty-interval trials (Experiment 2B). Unlike filled intervals, empty intervals require the processing of four events-that is, onsets and offsets of the auditory markers bounding the interval-whereas no signal is presented during the interval itself. If a more complex mode is more prone to error than the simplest mode, it should be the case that empty intervals are processed less accurately than filled intervals. A more complex switch mode may cause a misassignment of neural pulses generated by the internal pacemaker, and thus result in a performance decrement (Rammsayer \& Lima, 1991).

Because duration discrimination was found to be independent of signal energy in Experiment 1, the fact that filled intervals are more readily discriminated than empty intervals may be more readily explained in terms of differences in the structure of stimulus characteristics between filled and empty intervals than in terms of energydependent cues. Furthermore, the outcome of the present study shows that, even if the internal timing mechanism is more adapted to the processing of filled intervals than to the processing of empty intervals, even prolonged training will not be able to reduce the difference in durationdiscrimination performance between filled and empty intervals. In this respect, the finding of practice invariance points to the conclusion that the difference in durationdiscrimination performance observed with filled and empty intervals may be caused by different switch modes for processing of filled and empty intervals, and thus represents an essential feature of the internal timing mechanism.

Although the absence of practice effects might be unexpected from a psychological point of view (because most psychological functions are expected to improve with practice), from a pragmatic perspective, a clock or timing mechanism that was affected by various internal or external factors would not be a very reliable timepiece. That is, to ensure reliable timing, a timing mechanism has to maintain a stable clock rate despite possible interfering influences. This can be achieved by extensive protection from different interfering factors that may cause a slowing down in clock rate or an increase in variability of clock rate. In addition, an effective timing mechanism should run at an optimum clock rate to ensure the finest possible temporal resolution and, thus, the highest accuracy.
The lack of practice effects found in Experiments 2A and $2 \mathrm{~B}$ points to the existence of a timing mechanism that originally works at an optimum level. In this respect, the outcome of the present experiments suggests either a very basic biological timing mechanism that, unlike many cortical functions, is not susceptible to experience and training, or a highly overlearned perceptual process. The latter could result from the fine temporal resolution required for normal speech perception, particularly of voice-onset time (e.g., Eimas, Siqueland, Jusczyk, \& Vigorito, 1971; Lisker \& Abramson, 1967). However, in a study by Jusczyk, Pisoni, Reed, Fernald, and Myers (1983) on infants' discrimination of the duration of a rapid spectrum change in nonspeech signals, even 2-month-old infants were able to discriminate differences as small as $25 \mathrm{msec}$. This capacity may indicate the existence and the smooth functioning of an internal timing mechanism underlying temporal discrimination even in very young infants. Therefore, there is little reason to believe that the lack of practice effects can be interpreted in terms of a highly overlearned perceptual process underlying duration discrimination with brief auditory intervals in the range of milliseconds.

In an experiment on the effects of practice on dichotic sequence discrimination, Babkoff, Algom, and Eliasher (1980) studied detection of interaural temporal asymmetry by means of a three-alternative forced-choice procedure. In their study, the interval between dichotic clicks was varied from 4 to $64 \mathrm{msec}$. Continued practice over eight testing sessions did not result in either a systematic trend or any overall improvement. This finding also corroborates the proposition of a very basic biological process underlying temporal processing of brief auditory signals that is not influenced by extensive training.

In addition, the notion of a biological timing mechanism is supported by the outcome of pharmacopsychological studies on time perception. Mitrani, Shekerdjiiski, Gourevitch, and Yanev (1977) showed that LSD and mescaline, substances that strongly affect time estimation of longer intervals, did not alter duration discrimination of visually presented intervals in the range of milliseconds. On the basis of their results, Mitrani et al. concluded that short time intervals in the range of milliseconds are processed almost automatically at a lower level of the central nervous system and beyond cognitive control. Furthermore, in a series of experiments on the sedative effects of dopaminergic and serotonergic drugs as well as benzodiazepines on duration discrimination in the range of milliseconds, Rammsayer $(1989,1992 b)$ found duration discrimination to be independent of the effective level of vigilance and cortical arousal. This finding, too, suggests that the underlying timing mechanism is located at a subcortical rather than at a cortical level. Taken together, there is sufficient experimental evidence for the proposition of a very basic biological timing mechanism underlying temporal processing of stimuli in the range of milliseconds.

Although the present study strongly suggests that duration discrimination can be considered to be a function 
of the temporal extent of the stimulus and not of other stimulus parameters, it is important to note that the mean threshold value of $6.8 \mathrm{msec}$ computed across the 6 subjects of Experiment $2 \mathrm{~A}$ on the first testing day was substantially lower than the mean threshold value of 13.5 msec obtained in Experiment 1 under the S-C condition, with both the standard and comparison intervals presented at the same level of intensity. In previous studies from our laboratory (e.g., Rammsayer, 1992c; Rammsayer \& Lima, 1991) including more than 140 subjects, the resulting mean threshold value was $7.4 \mathrm{msec}$, which well agrees with the mean threshold value of $6.8 \mathrm{msec}$ observed in Experiment 2A. The difference in mean threshold values between Experiments 1 and 2A may represent an effect of stimulus context. For example, Penner (1976) found that randomizing intensity or duration of noise bursts bounding empty intervals resulted in a marked increase in threshold values as compared with fixed marker control conditions. The performance decrement appeared to be primarily due to the randomization of the duration of the first marker. Randomizing marker duration or marker intensity within a block of trials can be considered as an increase in task uncertainty, because subjects do not know the exact stimulus that will be presented in any given trial. However, when marker intensity was changed from block to block but held constant within each block, no such pronounced changes in discrimination performance could be observed (Carbotte \& Kristofferson, 1973). In Experiment 1, as in Penner's (1976) study, auditory intervals were presented at different levels of intensity in randomized order within one block, thus possibly inducing high stimulus uncertainty. Such a deleterious effect of stimulus uncertainty may point to higher level, nonperipheral effects that can interact with temporal information processing (Woods, Sorkin, \& Boggs, 1979).

In conclusion, the outcome of the present study did not prove that no energy-dependent cues are available, but rather that energy-dependent cues, if they exist, can be ignored. In this respect, temporal and energy-dependent cues represent separable, not integral, dimensions in Garner's (1974) sense. Duration discrimination of auditory intervals of less than $100 \mathrm{msec}$ appears to be based on an internal time code that is independent of signal energy. Furthermore, accuracy of temporal processing was not influenced by moderate training over 20 daily testing sessions. The absence of practice effects on duration discrimination with both filled and empty auditory intervals is in agreement with the proposition of a very basic biological timing mechanism underlying temporal processing that runs at a constant clock rate.

\section{REFERENCES}

AneL, S. M. (1972a). Discrimination of temporal gaps. Journal of the Acoustical Society of America, 52, 519-524.

ABEL, S. M. (1972b). Duration discrimination of noise and tone bursts. Journal of the Acoustical Society of America, 51, 1219-1223.
Allan, L. G. (1979). The perception of time. Perception \& Psychophysics, 26, 340-354

Allan, L. G., \& KrJstofferson, A. B. (1974a). Judgments about the duration of brief stimuli. Perception \& Psychophysics, 15, 434-440.

Allan, L. G., \& Kristofferson, A. B. (1974b). Psychophysical theories of duration discrimination. Perception \& Psychophysics, 16, 26-34.

Allan, L. G., Kristofferson, A. B., \& Wiens, E. W. (1971). Duration discrimination of brief light flashes. Perception \& Psychophysics, 9, 327-334.

Babkoff, H., Algom, D., \& Eliasher, S. (1980). Dichotic sequence discrimination: The effect of continued practice. Psychological Research, 42, 319-326.

BLock, R. A. (1990). Cognitive models of psychological time. Hillsdale, NJ: Erlbaum.

Carbotte, R. M., \& Kristofferson, A. B. (1973). On energydependent cues in duration discrimination. Perception \& Psychophysics, 14, 501-505.

Church, R. M. (1984). Properties of the internal clock. In J. Gibbon \& L. G. Allan (Eds.), Timing and time perception (Annals of the New York Academy of Sciences, Vol. 423, pp. 566-582). New York: New York Academy of Sciences.

COHEN, I. (1977). Statistical power analysis for the behavioral sciences. New York: Academic Press.

Craig, J. C. (1973). A constant error in the perception of brief temporal intervals. Perception \& Psychophysics, 13, 99-104.

Creelman, C. D. (1962). Human discrimination of auditory duration. Journal of the Acoustical Society of America, 34, 582-593.

Cronbach, L. J. (1990). Essentials of psychological testing. New York: Harper \& Row.

Divenyi, P. L., \& Danner, W. F. (1977). Discrimination of time intervals marked by brief acoustic pulses of various intensities and spectra. Perception \& Psychophysics, 21, 125-142.

Divenyi, P. L., \& SACHS, R. M. (1978). Discrimination of time intervals bounded by tone bursts. Perception \& Psychophysics, 24, 429-436.

Eimas, P. D., Siqueland, E. R., JusczyK, P., \& Vigorito, J. (1971). Speech perception in infants. Science, 171, 303-306.

Ekman, G., Berglund, B., \& Berglund, U. (1966). Loudness as a function of the duration of auditory stimulation. Scandinavian Journal of Psychology, 7, 201-208.

Fidell, S., Pearsons, K. S., Grignetti, M., \& Green, D. M. (1970). The noisiness of impulsive sounds. Journal of the Acoustical Society of America, 48, 1304-1310.

GARNER, W. R. (1949). The loudness and loudness matching of short tones. Journal of the Acoustical Society of America, 21, 398-403.

GARNER, W. R. (1974). The processing of information and structure. Potomac, MD: Erlbaum.

Garner, W. R., \& Miller, G. A. (1947). Differential sensitivity as a function of the duration of the comparison tone. Journal of Experimental Psychology, 34, 450-463.

GETTY, D. J. (1975). Discrimination of short temporal intervals: A comparison of two models. Perception \& Psychophysics, 18, 1-8.

HENRY, F. M. (1948). Discrimination of the duration of a sound. Journal of Experimental Psychology, 38, 734-743.

Jusczyk, P. W., Pisoni, D. B., Reed, M. A., Fernald, A., \& MYERS, M. (1983). Infants' discrimination of the duration of rapid spectrum change in nonspeech signals. Science, 222, 175-176.

KeLLY, D. R. (1972). Flicker. In D. Jameson \& L. M. Hurvich (Eds.), Visual psychophysics (pp. 273-302). Berlin: Springer-Verlag.

Keselman, H. J., \& Rogan, J. C. (1980). Repeated measures $F$ test and psychophysiological research: Controlling the number of false positives. Psychophysiology, 17, 499-503.

KRISTOFFERSON, A. B. (1980). A quantal step function in duration discrimination. Perception \& Psychophysics, 27, 300-306.

KRISTOFFERSON, A. B. (1984). Quantal and deterministic timing in human duration discrimination. In J. Gibbon \& L. G. Allan (Eds.), Timing and time perception (Annals of the New York Academy of Sciences, Vol. 423, pp. 3-15). New York: New York Academy of Sciences.

LEVITT, H. (1971). Transformed up-down methods in psychoacoustics. Journal of the Acoustical Society of America, 49, 467-477. 
LIPSEY, M. W. (1990). Design sensitivity: Statistical power for experimental research. Newbury Park, CA: Sage.

LiSkER, L., \& ABramson, A. S. (1967). Some effects of context on voice onset time in English stops. Language \& Speech, 10, 1-28.

Miller, G. A. (1948). The perception of short bursts of noise. Journal of the Acoustical Society of America, 20, 160-170.

Mitrani, L., Shekerdilski, S., Gourevitch, A., \& Yanev, S. (1977). Identification of short time intervals under LSD-25 and mescaline. Activitas Nervosa Superior, 19, 103-104.

Munson, W. A. (1947). The growth of auditory sensation. Journal of the Acoustical Society of America, 19, 384-391.

PenNer, M. J. (1975). Persistence and integration: Two consequences of a sliding integrator. Perception \& Psychophysics, 18, 114-120.

PenNer, M. J. (1976). The effect of marker variability on the discrimination of temporal intervals. Perception \& Psychophysics, 19, 466-469.

Rammsayer, T. (1989). Dopaminergic and serotoninergic influence on duration discrimination and vigilance. Pharmacopsychiatry, 22 (Suppl. 1), 39-43.

RAMMSAYER, T. (1990). Temporal discrimination in schizophrenic and affective disorders: Evidence for a dopamine-dependent internal clock. International Journal of Neuroscience, 53, 111-120.

RAMMSAYER, T. (1992a). Duration discrimination in the blind and nonblind: Evidence for a biological timing mechanism. Kognitionswissenschaft, 2, 180-188.

RAMMSAYER, T. (1992b). Effects of benzodiazepine-induced sedation on temporal processing. Human Psychopharmacology, 7, 311-318.

RammsaYer, T. (1992c). Die Wahrnehmung kurzer Zeitdauern [Perception of short durations]. Münster, Germany: Waxmann.

RAMMSAYER, T. H., \& LIMA, S. D. (1991). Duration discrimination of filled and empty auditory intervals: Cognitive and perceptual factors. Perception \& Psychophysics, 50, 565-574.

Rammsayer, T. H., \& Lustnauer, S. (1989). Sex differences in time perception. Perceptual \& Motor Skills, 68, 195-198.

Rousseau, R., \& Kristofferson, A. B. (1973). The discrimination of bimodal temporal gaps. Bulletin of the Psychonomic Society, 1 , 115-116.

Small, A. M., Brandt, J. F., \& Cox, P. G. (1962). Loudness as a function of signal duration. Journal of the Acoustical Society of America, 34, 513-514.

Small, A. M., \& CAMPBell, R. A. (1962). Temporal differential sensitivity for auditory stimuli. American Journal of Psychology, 75, 401-410.

Stevens, J. C., \& Hall, J. W. (1966). Brightness and loudness as functions of stimulus duration. Perception \& Psychophysics, 1, 319-327.

STEvens, S. S. (1966). Duration, luminance, and the brightness exponent. Perception \& Psychophysics, 1, 96-100.

Treisman, M. (1963). Temporal discrimination and the indifference interval: Implications for a model of the 'internal clock'. Psychological Monographs, 77, 1-31.

Treisman, M., Faulkner, A., Naish, P. L. N., \& Brogan, D. (1990). The internal clock: Evidence for a temporal oscillator underlying time perception with some estimates of its characteristic frequency. Perception, 19, 705-743.

Woods, D. D., SorkIN, R. D., \& Boggs, G. J. (1979). Stimulus context and duration discrimination. Perception \& Psychophysics, 26, 127-132.

(Manuscript received January 25, 1993; revision accepted for publication August 4, 1993.) 